\title{
Dynamics of Relativistic Solitons
}

\author{
Daniela Farina† and Sergei V. Bulanov $\$ \S$ \\ † Istituto di Fisica del Plasma "Piero Caldirola", Consiglio Nazionale delle \\ Ricerche, EURATOM-ENEA-CNR Association, Milan, Italy \\ $\ddagger$ Advanced Photon Research Center, Kansai Research Establishment, JAERI, \\ Kizu, Kyoto 619-0215, Japan \\ $\S$ A. M. Prokhorov Institute of General Physics, Russ. Acad. Sci., Moscow, \\ Russia
}

\begin{abstract}
Relativistic solitons are self-trapped, finite size, electromagnetic waves of relativistic intensity that propagate without diffraction spreading. They have been predicted theoretically within the relativistic fluid approximation, and have been observed in multi-dimensional particle in cell simulations of laser pulse interaction with the plasma. Solitons were observed in the laser irradiated plasmas with the proton imaging technique as well. This paper reviews many theoretical results on relativistic solitons in electron-ion plasmas.
\end{abstract}

\section{Introduction}

Relativistic solitons are self-trapped, finite size, electromagnetic waves of relativistic intensity $(e E / m \omega c \geq 1)$ that propagate without diffraction spreading. Many different physical effects play a role in the formation of relativistic solitons: dispersion effects due to the finite particle inertia, nonlinearities due to relativistic mass increase, as well as ponderomotive effects which force the plasma density redistribution.

The theoretical investigation of relativistic solitons in electron-ion plasmas is a relatively old problem in plasma physics, which has been treated by many authors in the past, and has recently gained new attention in the literature 1, 2, 3, 4, 5, 6, 7, 8, 9, 10, 11, 12, 13, 14, 15, 16. The analyses have been performed mainly in the framework of the 1D relativistic fluid approximation, in which solitons are described by the solutions of a set of coupled nonlinear differential equations for the electrostatic and electromagnetic potentials with suitable boundary conditions.

Relativistic solitons have been seen in multi-dimensional particle in cell (PIC) as well as in fluid simulations of laser pulse interaction with the plasma 17, 18, 19, 20, 21, 22, 23, 24. These solitons consist of electron density depressions and intense electromagnetic field concentrations with a larger amplitude and a lower frequency than those of the laser pulse. Since a significant portion of the overall electromagnetic energy is trapped in the form of solitons, solitary waves can play an important role in the laser-plasma interaction.

\section{1D relativistic solitons}

Here, the theory of 1D circularly polarized solitons is presented within the relativistic hydrodynamic approximation used to describe both the electron and ion components 
[5. 10, 11. The plasma is assumed to be cold with zero ion and electron temperature. Length, time, velocity, momentum, vector and scalar potential, and density are normalized over $c / \omega_{p e}, \omega_{p e}, c, m_{\alpha} c, m_{e} c^{2} / e$, and $n_{0}$, respectively, being $\omega_{p e}=$ $\left(4 \pi n_{0} e^{2} / m_{e}\right)^{1 / 2}$ the electron plasma frequency, $m_{\alpha}$ the rest mass with $\alpha=e, i$, and $n_{0}$ the unperturbed electron (and ion) density. In the Coulomb gauge, the Maxwell's equations for the vector and scalar potentials, $\mathbf{A}$ and $\phi$, and the hydrodynamic equations (continuity, and momentum balance) for the densities $n_{\alpha}$ and the canonical momentum $\mathbf{P}_{\alpha}$ of electrons and ions can be written as

$$
\begin{aligned}
& \triangle \mathbf{A}-\frac{\partial^{2}}{\partial t^{2}} \mathbf{A}-\frac{\partial}{\partial t} \nabla \phi=n_{e} \mathbf{v}_{e}-n_{i} \mathbf{v}_{i}, \\
& \triangle \phi=n_{e}-n_{i}, \\
& \frac{\partial}{\partial t} n_{\alpha}+\nabla \cdot\left(n_{\alpha} \mathbf{v}_{\alpha}\right)=0, \\
& \frac{\partial}{\partial t} \mathbf{P}_{\alpha}=-\nabla\left(\rho_{\alpha} \phi+\gamma_{\alpha}\right)+\mathbf{v}_{\alpha} \times \nabla \times \mathbf{P}_{\alpha},
\end{aligned}
$$

where $\mathbf{P}_{\alpha}$, and $\gamma_{\alpha}$ are related to the kinetic momentum $\mathbf{p}_{\alpha}$ by $\mathbf{P}_{\alpha}=\mathbf{p}_{\alpha}+\rho_{\alpha} \mathbf{A}$, and $\gamma_{\alpha}=\left(1+\left|\mathbf{p}_{\alpha}\right|^{2}\right)^{1 / 2}$ with the parameter $\rho_{\alpha}=\left(q_{\alpha} / q_{e}\right)\left(m_{\alpha} / m_{e}\right)$, and $\mathbf{v}_{\alpha}=\mathbf{p}_{\alpha} / \gamma_{\alpha}$ is the fluid velocity.

For the $1 \mathrm{D}$ case in which $\partial_{y}=\partial_{z}=0$, the vector potential is assumed of the form (circular polarized wave)

$$
A_{y}+i A_{z}=a(\xi) \exp [i \theta(\xi)] \exp (-i \omega t+i k x)
$$

with $\xi=x-V t$, while all the other quantities, $\phi, n_{\alpha}, \gamma_{\alpha}$, and $p_{x \alpha}$, are assumed to depend only on the variable $\xi$. In the present case, the relations $A_{x}=0$ and $\mathbf{P}_{\perp}=0$ are satisfied, where the symbol $\perp$ refers to the direction of the laser pulse propagation.

Imposing as boundary condition at the point $\xi=-\infty, a= \pm a_{0}, \phi=0$, $n_{\alpha}=1$, and $p_{x \alpha}=0$ (plasma at rest), the longitudinal component of the kinetic momentum, the energy, and the density of each species can be expressed as a function of the potentials as $p_{x \alpha}=\left(V \psi_{\alpha}-R_{\alpha}\right) /\left(1-V^{2}\right), \gamma_{\alpha}=\left(\psi_{\alpha}-V R_{\alpha}\right) /\left(1-V^{2}\right)$, $n_{\alpha}=V\left(\psi_{\alpha} / R_{\alpha}-V\right) /\left(1-V^{2}\right)$, where $\psi_{\alpha}=\Gamma_{0 \alpha}+\rho_{\alpha} \phi, R_{\alpha}=\left[\psi_{\alpha}^{2}-\left(1-V^{2}\right) \Gamma_{\alpha}^{2}\right]^{1 / 2}$, $\Gamma_{\alpha}=\sqrt{1+\rho_{\alpha}^{2} a^{2}}$ and $\Gamma_{0 \alpha}=\sqrt{1+\rho_{\alpha}^{2} a_{0}^{2}}$. Then, for an electron-ion plasma the following closed system of equations for the potentials is obtained

$$
\begin{aligned}
& \frac{d^{2} \phi}{d \xi^{2}}=\frac{V}{1-V^{2}}\left(\frac{\psi_{e}}{R_{e}}-\frac{\psi_{i}}{R_{i}}\right), \\
& \frac{d^{2} a}{d \xi^{2}}+a\left(\bar{\omega}^{2}-\bar{k}^{2} \frac{a_{0}^{4}}{a^{4}}\right)=a \frac{V}{1-V^{2}}\left(\frac{1}{R_{e}}+\frac{\rho}{R_{i}}\right) . \\
& \frac{d \theta}{d \xi}=-\bar{k}\left(1-\frac{a_{0}^{2}}{a^{2}}\right)
\end{aligned}
$$

where $\bar{\omega}=(\omega-k V) /\left(1-V^{2}\right), \bar{k}=(k-\omega V) /\left(1-V^{2}\right)$, and $\rho \equiv\left|\rho_{i}\right|=m_{e} / m_{i}$.

The system of equations (677) describes coupled Langmuir and circularly polarized transverse electromagnetic waves. Note that the r.h.s. of (7) is proportional to $n_{e} / \gamma_{e}-n_{i} / \gamma_{i}$ and thus represents the balance between the striction nonlinearity due to perturbation of the density and the relativistic nonlinearity due to mass. Equation (8) describes the related phase evolution of the e. m. amplitude. For $a_{0}=0$, the solution of (8) is simply $\theta=\theta_{0}-\bar{k} \xi$, so that the e. m. field defined in (5D) has the simple form $a(\xi) \exp \left[-i \bar{\omega} \tau+i \theta_{0}\right]$, being $\tau=t-V x$. For $a_{0} \neq 0$, the phase evolution is non trivial. 
The system (6.7) can be put in Hamiltonian form, and has a first integral

$$
H\left(a, a^{\prime}, \phi, \phi^{\prime}\right)=\frac{1-V^{2}}{2}\left(a^{\prime 2}+\bar{\omega}^{2} a^{2}+\bar{k}^{2} \frac{a_{0}^{4}}{a^{2}}\right)-\frac{1}{2} \phi^{\prime 2}-\gamma_{e}-\frac{\gamma_{i}}{\rho}=K(9)
$$

where the symbol / denotes derivative with respect to $\xi$. The value of the constant $K$ is determined by the boundary condition.

For the case of a pure transverse electromagnetic wave (i.e., $\phi=0$ and $a=a_{0}$ ), from (7) the dispersion equation is obtained

$$
\omega^{2}=\Omega^{2}+k^{2}, \quad \Omega^{2}=\frac{1}{\sqrt{1+a_{0}^{2}}}+\frac{\rho}{\sqrt{1+\rho^{2} a_{0}^{2}}}
$$

$\Omega$ being the plasma frequency modified by the relativistic effects. Equation (10) corresponds to the Akhiezer-Polovin result [25] with the ion motion taken into account.

So far, explicit reference to an electron-ion plasma has been made. However, putting $\rho=1$, Eqs. (6.9) are valid also in a electron-positron plasma [26].

The particular class of localized solutions of equations (6.7) is investigated, satisfying $a^{\prime}=0, a^{\prime \prime}=0, \phi^{\prime}=0$, and $\phi^{\prime \prime}=0$ at $\xi= \pm \infty$. For sake of simplicity, the case $\bar{k}=0$ is considered, so that $k=\omega V$, and $\bar{\omega}=\omega$ (see e.g., [5]). Note that here $V$ is the group velocity.

\subsection{Quasineutral approximation}

Within the quasineutral approximation the main features of the localized solutions can be identified in a simple manner, assuming $n_{e}-n_{i}$, i.e., putting $\phi^{\prime \prime}=0$ [5]. Then, the electrostatic potential can be written in terms of the vector potential amplitude, $\phi=\left(\Gamma_{0 i} \Gamma_{e}-\Gamma_{0 e} \Gamma_{i}\right) / \Gamma$, being $\Gamma=\Gamma_{i}+\rho \Gamma_{e}$, and the equation for the vector potential in the quasineutral approximation becomes [1]

$$
a^{\prime \prime}+\bar{\omega}^{2} a=a \frac{V}{1-V^{2}} \frac{\Gamma^{2}}{\Gamma_{i} \Gamma_{e}\left[\Gamma_{0}^{2}-\left(1-V^{2}\right) \Gamma^{2}\right]^{1 / 2}} .
$$

The above equation has the following first integral $H_{0}\left(a, a^{\prime}\right)=1 / 2\left(a^{\prime 2}+\bar{\omega}^{2} a^{2}\right)+$ $V \sqrt{\Gamma_{0}^{2}-\left(1-V^{2}\right) \Gamma^{2}} / \rho\left(1-V^{2}\right)^{2}=K_{0}$, and the solution for $a$ is reduced to quadrature. Then, in the limit $\rho \ll 1$, the following simple expressions hold: $\phi \approx \sqrt{1+a^{2}}-\sqrt{1+a_{0}^{2}}, n_{e}=n_{i} \approx 1+\rho \phi / V^{2}$, and $v_{x e, i} \approx \rho \phi / V$.

From the analysis of the topology of the phase space $\left(a, a^{\prime}\right)$ of the Hamiltonian $H_{0}$, different kind of solitary solutions are found varying the propagation speed, and are summarized in table 1

Table 1. Solitons in a cold plasma.

\begin{tabular}{lllll}
\hline $\begin{array}{l}\text { Propagation } \\
\text { speed }\end{array}$ & $\begin{array}{l}\text { Solution } \\
\text { kind }\end{array}$ & $\begin{array}{l}\text { Potential } a \\
\text { at }-\infty,+\infty\end{array}$ & $\begin{array}{l}\text { Potential } \phi \\
\text { profile }\end{array}$ & $\begin{array}{l}\text { Density } \\
\text { profile }\end{array}$ \\
\hline$V^{2}>V_{s 1}^{2}$ & bright & 0,0 & positive & accumulation \\
$V^{2}=V_{s}^{2}$ & shock wave & $0, a_{1}$ & positive & compression \\
$V_{s}^{2}<V^{2}<V_{c}^{2}$ & dark (gray) & $a_{0}, a_{0}$ & negative & evacuation \\
$V^{2}=V_{s}^{2}$ & shock wave & $a_{0}, 0$ & negative & rarefaction \\
$0<V^{2}<V_{s}^{2}$ & dark (black) & $a_{0},-a_{0}$ & negative & evacuation \\
\hline
\end{tabular}

Solitary solutions are found for $\omega^{2}\left(1-V^{2}\right)<1+\rho$, with dark solitons satisfying the dispersion relation (10). In both cases, the frequency spectrum corresponds to the 
evanescent region in the $(\omega, k)$ space, i.e., to the region $\omega^{2}-k^{2}<1+\rho$. When the ion dynamics is taken into account, bright solitons occurs for propagation speed larger than a threshold value scaling with $\sqrt{\rho}$, and dark solitons occur below this threshold value. Standing bright solitons are found only when the ion dynamics is neglected. Solutions in the form of shock-waves are found at critical propagation speeds. These shock waves (occurring in the cold plasma limit) are collisionless, and similar to those found at ion-acoustic speed [27]. The characteristic velocities introduced in table 1 depend on the potential amplitude and have the following expressions in the low amplitude limit and for $\rho \ll 1: V_{s 1}^{2} \approx \rho\left(1+a_{1}^{2}\right), V_{s}^{2} \approx \rho\left(1+a_{0}^{4} / 16\right)$, $V_{c}^{2} \approx \rho\left(1+a_{0}^{2} / 2\right)$. In the same limit, bright solitons are described by $a=a_{m} \operatorname{sech}(\kappa \xi)$, with $\kappa^{2}=1+\rho-\omega^{2}\left(1-V^{2}\right)$, and $a_{m}^{2}=4 \kappa^{2} /\left(1-\rho / V^{2}\right)$, while dark solitons by $a=a_{0} \tanh (\kappa \xi)$, with $a_{0}^{2}=4 \kappa^{2} /\left(\rho / V^{2}-1\right)$, and $\omega^{2}\left(1-V^{2}\right)=1+\rho-a_{0}^{2} / 2$ [5] [1].

At large amplitude, quasineutrality is violated [5], and the set of coupled equations (617) has to be solved numerically. However, the above investigation on the existence of the different kind of solutions has a general validity.

\subsection{Bright solitons in e-i plasma}

In the case of large amplitude bright solitons, a wide class of solutions can be found. Here, the analysis is restricted to the class of solutions with single humped $\phi$ profiles, and $a$ profiles characterized by the number of nodes $p$. For fixed $V$, the eigenspectrum of the bright solitons can been determined solving numerically Eqs. (6 7) with suitable boundary conditions [5, 10].

The eigenspectrum relevant for low $p$ values is shown in figure 1 both for the case in which the ions are fixed, i.e., at $\rho=0$, and for the case in which the ion dynamics is taken into account.
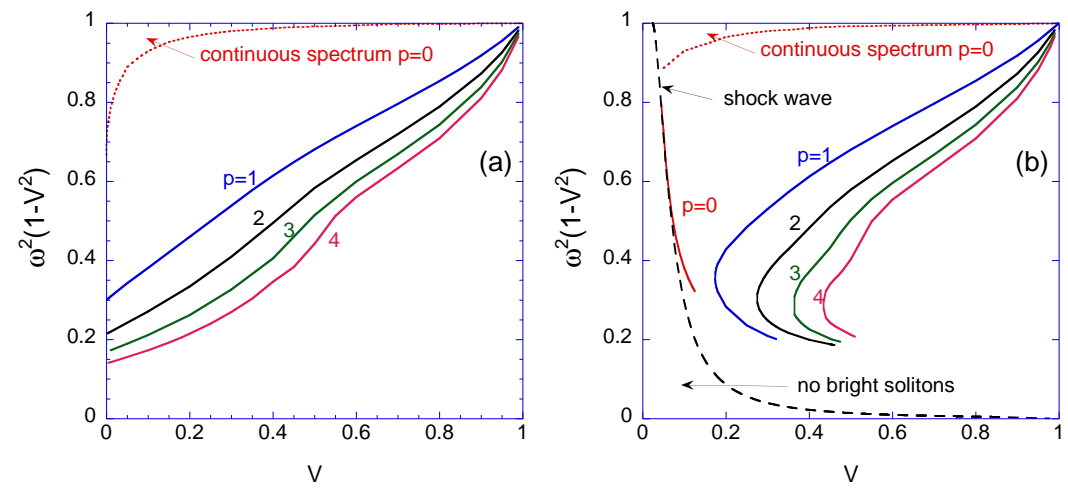

Figure 1. Eigenspectrum of bright solitons as a function of the group velocity $V$. Cases (a) and (b) refer to $\rho=0$ (no ion dynamics) and $\rho=1 / 1836$ (with ion dynamics), respectively. Both the regions corresponding to the continuos spectrum of single-peaked solitons and the discrete spectrum for $p=0, \ldots, 4$ are shown. The dashed curve in frame (b) delimits a region of no solutions.

When the ion dynamics is neglected (i.e., $\rho=0$ ), the bright soliton eigenspectrum has the following features. Single-peaked solitons $(p=0)$ admit a continuous spectrum for any velocity value [13]. In particular, standing stable solitons $(V=0)$ are found with frequency in the range $2 / 3 \leq \omega^{2}<1$, and $\max a \leq \sqrt{3}$, as it has been shown 
analytically in [9]. Multi-peaked solitons $(p \geq 1)$ have a discrete spectrum for velocities larger than a small critical value, and at fixed $V$, the frequency $\omega$ decreases with increasing $p$. The peak value of the potentials $a$ and $\phi$ is found at the minimum velocity, and increases with $p$ [11.

Quite different results are found when the ion dynamics is taken into account (i.e., $\rho \neq 0$ ), even at a finite propagation speed. Note that at high frequencies (and velocities), the ion dynamics does not play any role. Single-humped solutions are found for velocities larger than $V_{s} \approx \sqrt{\rho}[5$, with both a continuous spectrum [13] and a discrete spectrum [10]. For $p \geq 1$, only a discrete spectrum is found [10. At low $p$ values, the frequency is non monotonous as a function of $V$, and at $V=V_{b r}$ the branch ends since the soliton breaks. The structure of the solution for $p=0,1,2$ and a velocity value close to breaking is shown in figure 2 where the potential waveforms are plotted. It is found that the ions pile at the soliton center, while the electrons at its edges, giving rise to very peaked density distributions, and breaking occurs because the ion density diverges in the center. At the same time, the ion and electron velocities tend to $V_{b r}$ at the center of the soliton and at its edges, respectively, so that in these regions the particles move with almost the group velocity of the soliton. The ion velocity profile shows a cusp in the center, which is the signature of the nonlinear wavebreaking. The soliton breaking provides a novel mechanism for the ion acceleration in the high intensity laser pulse interaction with plasmas [10].

Solitons at large $p$ number have been investigated numerically and analytically in different approximations [6, 7, 8, 13].
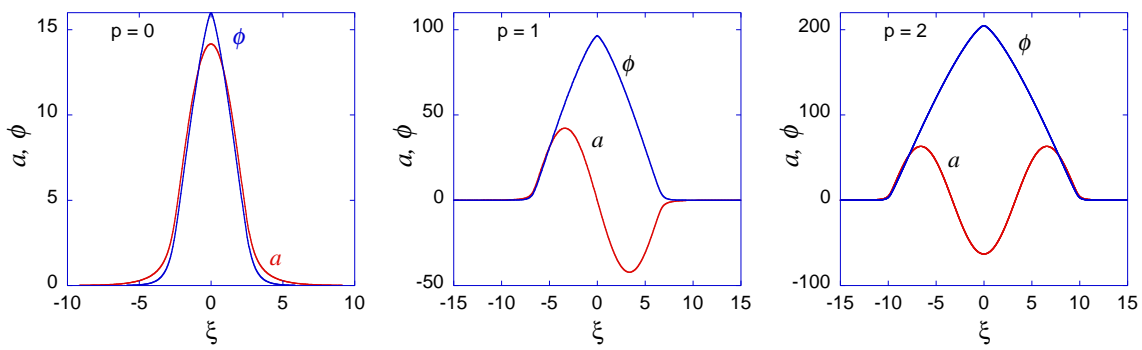

Figure 2. Potential waveforms for bright solitons with $p=0,1,2$ and velocities close to breaking.

\subsection{Dark solitons in e-i plasma}

As already pointed out, dark solitons are found only when the ion dynamics is taken into account and at very low velocities $(V \lesssim \sqrt{\rho})$. The solutions of the full nonlinear system for $a_{0} \neq 0$ are obtained numerically, as in the case of bright solitons [12. The obtained spectrum and the corresponding $a_{0}$ value are shown in figure 3 as a function of the velocity. At fixed $V$, the spectrum is continuous at low $a_{0}$, and it becomes discrete above a threshold value, which increases with $V$. The discrete spectrum is made up by a large number of separate branches, each of them characterized by different densities and velocities profiles. The ion density profile show always a dip, while at large $a_{0}$ the electron density tends to peak in the region inside the soliton. At a critical amplitude value (dependent on $V$ ) breaking of the solutions occurs, due to the peaking of the electron density. Solitary solutions are found up to $V \approx 0.051$, and 
$a_{0} \approx 5.8$ (for $\rho=1 / 1836$ ). Above these values, no solutions have been found for the chosen parameters. Both black and gray solitons are found for $V$ smaller and larger than $V_{s}$, while a shock wave is found at $V=V_{s}$. Figure 4 shows the waveforms of the potentials for dark solitons (black and gray) and the shock wave.
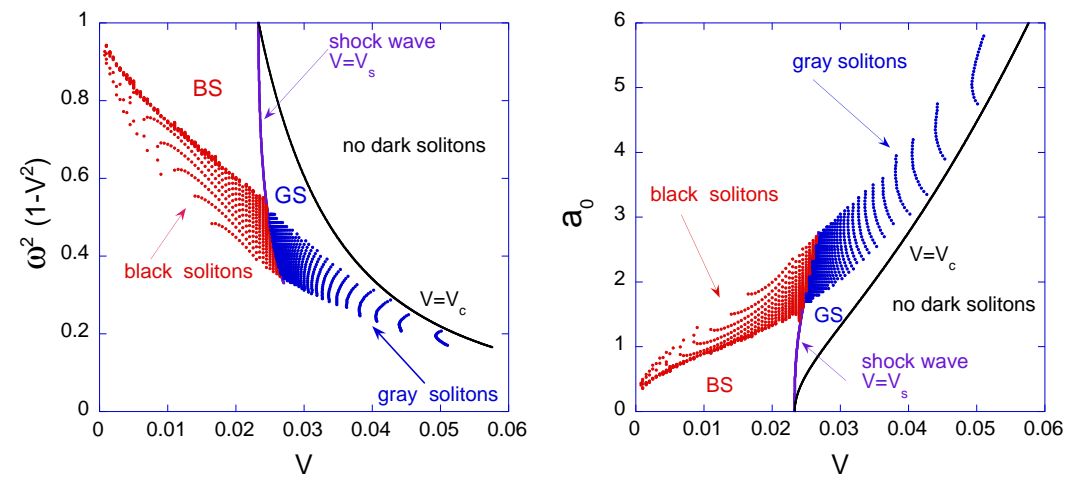

Figure 3. Eigenspectrum of dark solitons (a), and asymptotic e. m. amplitude $a_{0}$ (b) as a function of the group velocity $V$. The symbols GS and BS indicate the regions corresponding to the continuous spectrum of gray and black dark solitons, respectively. The curve corresponding to shock waves is also plotted.
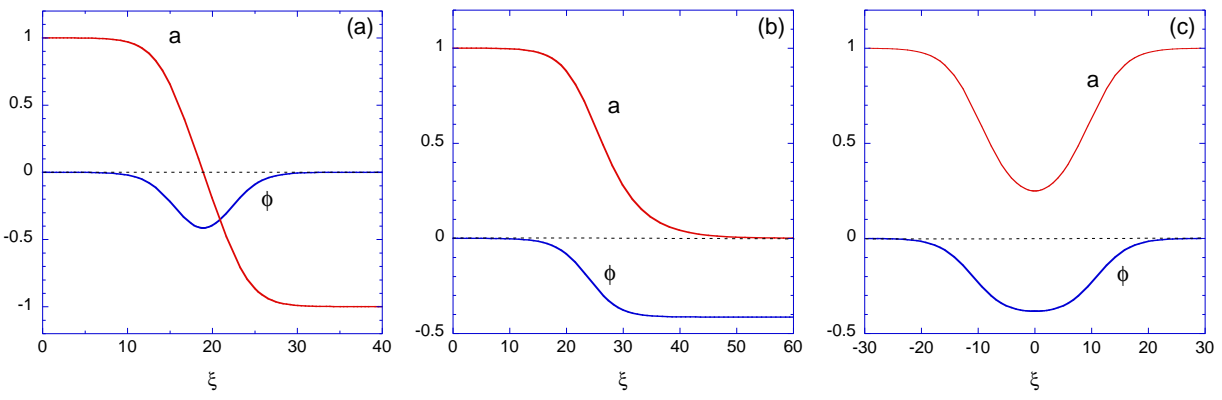

Figure 4. Waveforms of a black soliton (a), a shock wave (b) and a gray soliton (c). The chosen parameters are $a_{0}=1$, and $V<V_{s}, V=V_{s}$, and $V_{s}<V<V_{c}$, respectively.

\subsection{Warm plasma effects}

The results described in the previous section have been derived in the cold plasma approximation. However, it can be easily argued that solitons moving at "low" velocity can be affected by pressure terms. The theoretical investigation of temperature effects has been performed within the quasineutral approximation for standing solitons in a fully relativistic treatment [14], and for the case of moving solitons in the weakly relativistic approximation [15. The results are summarized in table 20 15, which refer to small finite amplitude solitons and non relativistic temperatures.

As expected, temperature effects play a crucial role in the range $0 \leq V^{2}<\rho+c_{s}^{2}$, being $c_{s}$ the ion-acoustic speed. Note that both inertia and temperature terms drive 
Table 2. Solitons properties in a warm plasma. The following symbols are used: $V_{t e, i}^{2}=T_{e, i} / m_{e, i} c^{2}, c_{s}^{2}=\Gamma_{e} \rho V_{t e}^{2}+\Gamma_{i} V_{t i}^{2}$, with $\Gamma_{e, i}$ the adiabaticity parameter.

\begin{tabular}{lllll}
\hline $\begin{array}{l}\text { Propagation } \\
\text { speed }\end{array}$ & $\begin{array}{l}\text { Soliton } \\
\text { variety }\end{array}$ & $\begin{array}{l}\text { Potential } \phi \\
\text { at the center }\end{array}$ & $\begin{array}{l}\text { Density } \\
\text { at the center }\end{array}$ & $\begin{array}{l}\text { Species } \\
\text { involved }\end{array}$ \\
\hline $0 \leq V^{2}<V_{t i}^{2}$ & bright & positive & evacuation & $\mathrm{i}+\mathrm{e}$ \\
$V_{t i}^{2}<V^{2}<c_{s}^{2}$ & bright & negative & $\begin{array}{l}\text { evacuation } \\
\text { evacuation }\end{array}$ & $\begin{array}{l}\mathrm{i}+\mathrm{e} \\
\mathrm{i}+\mathrm{e}\end{array}$ \\
$c_{s}^{2}<V^{2}<\rho+c_{s}^{2}$ & dark & negative & accumulation & $\mathrm{i}+\mathrm{e}$ \\
$\rho+c_{s}^{2}<V^{2}$ & bright & positive & evacuation & only e \\
$\rho+c_{s}^{2} \ll V^{2}$ & bright & positive & & \\
\hline
\end{tabular}

the process of soliton formation in a warm plasma. In particular, the formation of single-peaked solitons is observed at zero and very low propagation speed, lower than the ion acoustic speed. The relevant electron and ion density profiles show a dip in the center of the soliton. In the low temperature limit, density depletion in the center of standing solitons may become almost total [14].

\section{Solitons in laser plasma simulations}

Relativistic solitons has been investigated with the help of fluid simulations and 2D, 3D PIC simulations for laser-plasma interaction [17, 18, 19, 21, 22, 23. The mechanism of soliton generation by a high intensity laser pulse propagating in a underdense plasma can be descibed as follows. The laser pulse interacting with the plasma loses its energy, as it generates wake fields behind itself. Since the process is adiabatic the ratio between the e. m. energy density and the frequency is conserved, so that frequency downshift occurs for the wake field. Moreover, since the group velocity of the laser pulse decreases with the frequency, the low frequency part of the e. m. radiation of the pulse propagates with very low velocity. When the frequency becomes lower than the Langmuir frequency, the e. m. energy becomes trapped inside the related density cavity, thus forming a slowly moving solitary structure. Note that in $2 \mathrm{D}$ and $3 \mathrm{D}$ it is found that solitary waves can merge, thus they are not strictly speaking solitons. It has been observed that a large part of the laser pulse energy can be transformed into solitons [19. It can then be argued that solitary waves may play an important role in the laser-plasma interaction.

When PIC simulations are performed in inhomogeneous plasmas [20, it is found that low frequency solitary waves generated by superintense laser pulses are accelerated along the density gradient towards the lowest density values (e.g., the plasma-vacuum interface) where they radiate their energy in the form of low frequency electromagnetic bursts. This process can be a signature of soliton formation.

PIC simulations show that the time of the soliton formation is much shorter than the ion response time, so that ions can be assumed at rest during this process. For approximately $\left(m_{i} / m_{e}\right)^{1 / 2}$ oscillation periods of the e. $\mathrm{m}$. field inside the soliton (which is of the order of $2 \pi / \omega_{p e}$ ), the ions can be assumed as fixed. For longer times, the ponderomotive force starts to dig a hole in the ion density, and the parameters of the solitons change. What was a soliton on the electron timescale is no more a soliton on the ion timescale. Nevertheless, a low frequency e. m. wave packet remains well confined inside a slowly expanding plasma cavity. This e. m. entity has been called a postsoliton 21]. 
In addition, 1D fluid simulations in "slightly" overdense plasmas (i.e., for plasma densities $\lesssim 1.5$ times the critical density) have shown that penetration of relativistically intense laser radiation occurs by soliton-like structures moving into the plasma 24.

\section{Final remarks}

Many theoretical problems are still open even for 1D solitons, as, e.g., the temperature effects at any propagation speed and arbitrary wave amplitude, the soliton dynamics in the presence of plasma inhomogeneity, the properties of linearly polarized solitons, and last but not least the stability of such structures (e. g., see discussions in Ref. [16]).

In conclusion, we point out that the first macroscopic evidence of soliton formation in the interaction of an intense $\left(10^{19} \mathrm{~W} / \mathrm{cm}^{2}\right)$ laser pulse with an underdense plasma has been reported in 28. Long-lived, macroscopic bubble-like structures have been detected through the deflection that the associated electric charge separation causes in a proton probe beam 29. These structures are interpreted as the remnants of a cloud of relativistic solitons generated in the plasma by the ultraintense laser pulse.

\section{References}

[1] Gerstein J I and Tzoar N 1975 Phys. Rev. Lett. 35934

[2] Marburger J H and Tooper R F 1975 Phys. Rev. Lett. 351001

[3] Lai C S 1976 Phys. Rev. Lett. 36966

[4] Tsintsadze N L and Tskhakaya D D 1977 Sov. Phys. JETP 45252

[5] Kozlov V A, Litvak A G, and Suvorov E V 1979 Sov. Phys. JETP 4975

[6] Kaw P K, Sen A and Katsouleas T 1992 Phys. Rev. Lett. 683172

[7] Kuehl H H and Zhang C H 1993 Phys. Rev. E 481316

[8] Sudan R N, Dimant Ya S and Shiryaev O B 1997 Phys. Plasmas 41489

[9] Esirkepov T Zh, Kamenets F F, Bulanov S V and Naumova N M 1998 JETP Lett. 6836

[10] Farina D and Bulanov S V 2001 Phys. Rev. Lett. 865289

[11] Farina D and Bulanov S V 2001 S V Plasma Phys. Rep. 27641

[12] Farina D and Bulanov S V 2002 AIP Conf. Proc. 611151

[13] Poornokala S, Das A, Sen A and Kaw P K 2002 Phys. Plasma 91820

[14] Lontano M, Passoni M and Bulanov S V 2003 Phys. Plasma 10639

[15] Poornokala S, Das A, Kaw P K, Sen A, Sheng Z M, Sentoku Y, Mima K and Nishikawa K 2002 Phys. Plasma 93802

[16] Hadžievski Lj, Jovanović M S, Škorić M M and Mima K, 2002 Phys. Plasma 92569

[17] Bulanov S V, Inovenkov I N, Kirsanov V I, Naumova N M and Sakharov A S 1992 Phys. Fluids B 41935

[18] Bulanov S V, Kamenetes F F, Esirkepov T Zh and Naumova N M 1995 Plasma Phys.Rep. 21 550

[19] Bulanov S V, Esirkepov T Zh, Naumova N M, Pegoraro F and Vshivkov V A 1999 Phys. Rev. Lett. 823440

[20] Sentoku Y et al 1999 Phys. Rev. Lett. 833434

[21] Naumova N M, Bulanov S V, Esirkepov T Zh, Farina D, Nishihara K, Pegoraro F, Ruhl H and Sakharov A S 2001 Phys. Rev. Lett. 87185004

[22] Esirkepov T Zh, Nishihara K, Bulanov S V and Pegoraro F 2002 Phys. Rev. Lett. 89275002

[23] Esirkepov T Zh, Bulanov S V, Nishihara K and Tajima T 2004 Phys. Rev. Lett. 92255001

[24] Tushentsov M, Kim A, Cattani F, Anderson D and Lisak M 2001 Phys. Rev. Lett. 87275002

[25] Akhiezer A I and Polovin R V 1956 Sov Phys. JETP 30915

[26] Farina D and Bulanov S V 2001 Phys. Rev. E 64066401

[27] Mima K, Nishikawa K and Ikezi H. 1975 Phys. Rev. Lett. 35726

[28] Borghesi M et al 2002 Phys. Rev. Lett. 88135002

[29] Borghesi M et al 2002 Phys. Plasma 92214 